\title{
The Configuration Formation of Planetary Systems Observed by Kepler
}

\author{
Su Wang and Jianghui Ji \\ Purple Mountain Observatory, Chinese Academy of Sciences, \\ Nanjing 210008, China \\ email: wangsu@pmo.ac.cn
}

\begin{abstract}
The Kepler mission has found many planetary systems, among them more than 80 systems host three planet candidates which reveal a configuration of near 4:2:1 mean motion resonance. In this paper, we focus on the configuration formation of resonant systems. As shown from our model and $\mathrm{N}$-body simulations, we find that 3:2 mean motion resonance always forms at the early stage of star evolution and planets undergo high rate of migration, while 2:1 mean motion resonance happens at the late stage of the star formation, more often.
\end{abstract}

Keywords. (stars:) planetary systems: formation

\section{Introduction}

The Kepler Mission, which can detect terrestrial planets in habitable zone, has released their 16 months data (Batalha et al. 2012; Fabrycky et al. 2012). According to the statistical results on the 2321 planet candidates, about $20 \%$ of them are in multiple planetary systems and more than $16 \%$ multiple systems contain a planet pair close to 2:1 mean motion resonance (MMR) (Period ratio of two planets is in the range of 1.83 to 2.18, Lissauer et al. 2011). Fabrycky et al. (2012) plot the distribution of the period ratios of all planet pairs in all candidate systems and find that the number of the planet pair close to 1.5 is higher than that close to 2 . We study the period ratio of planet pair in multiple planetary systems with three planets, especially those for which the period ratio of planet pairs closed to 2 is higher than 1.5 for the inner two planets, while it is opposite for the outer two planets.

Based on the analysis of five Kepler candidate exoplanetary systems, Steffen et al. (2010) found that KOI-152 (KOI: Kepler Objects of Interest) is a potential resonant system with three planets in 4:2:1 MMRs. There are about 10 systems with similar configurations. Table 1 shows the orbital period of each planet and the period ratio of the planet pair. Additionally, Table 1 shows five systems with planets potentially in 3:2:1 MMRs whose outer two planets are in 3:2 MMR instead of 2:1 MMRs as in 4:2:1 MMRs. Studying the configuration formation of these resonant systems is of value to understanding the formation and evolution of exoplanetary systems. In this paper, we simulate the configuration formation of the system KOI-152 and the Solar-like planetary systems.

\section{Formation scenario and N-body simulation results}

From Table 1 we see that planets are very close to the central body with short orbital periods. Based on the core-accretion model, such configuration can be formed in three steps. First, three planets formed far from the central star where they can grow up to the nominal masses with more solid materials there. Then, due to the interaction with the 
Table 1. The orbital periods of each planetary candidate and their ratios. Quantities $P_{01}$, $P_{02}, P_{03}, P_{04}$ and $P_{05}$ are the orbital periods of the planets in the systems.

\begin{tabular}{llllllllll}
\hline ID & $\begin{array}{l}P_{01} \\
\text { KOI }\end{array}$ & $\begin{array}{l}P_{02} \\
\text { (days) }\end{array}$ & $\begin{array}{l}P_{03} \\
\text { (days) }\end{array}$ & $\begin{array}{l}P_{04} \\
\text { (days) }\end{array}$ & $\begin{array}{l}P_{05} \\
\text { (days) }\end{array}$ & $P_{01} / P_{02}$ & $P_{02} / P_{03}$ & $P_{03} / P_{04}$ & $P_{04} / P_{05}$ \\
\hline 152 & 52.09119 & 27.40415 & 13.484 & - & - & 1.9009 & 2.0323 & - & - \\
571 & 13.34331 & 7.26733 & 3.886758 & - & - & 1.8361 & 1.8698 & - & - \\
665 & 5.867973 & 3.07154 & 1.611912 & - & - & 1.9104 & 1.9055 & - & - \\
733 & 11.34917 & 5.924992 & 3.132968 & - & - & 1.9155 & 1.8912 & - & - \\
829 & 38.5596 & 18.64902 & 9.75222 & - & - & 2.0676 & 1.9123 & - & - \\
898 & 20.08923 & 9.77059 & 5.16991 & - & - & 2.0561 & 1.8899 & - & - \\
899 & 15.36813 & 7.11388 & 3.306569 & - & - & 2.1603 & 2.1514 & - & - \\
1426 & 150.0341 & 74.91443 & 38.87641 & - & - & 2.0027 & 1.9270 & - & - \\
1860 & 12.2094 & 6.3194 & 3.0765 & - & - & 1.9321 & 2.0541 & - & - \\
1895 & 32.1349 & 17.2812 & 8.4575 & - & - & 1.8595 & 2.0433 & - & - \\
935 & 87.6464 & 42.6329 & 20.85987 & 9.6168 & - & 2.0558 & 2.0438 & 2.1691 & - \\
620 & 130.1831 & 85.3129 & 45.1555 & - & - & 1.5259 & 1.8893 & - & - \\
623 & 15.6775 & 10.3497 & 5.5994 & - & - & 1.5148 & 1.8484 & - & - \\
934 & 18.7466 & 12.4121 & 5.8267 & - & - & 1.5103 & 2.1302 & - & - \\
1127 & 8.1281 & 5.3267 & 2.8368 & - & - & 1.5259 & 1.8777 & - & - \\
1589 & 44.5462 & 27.4348 & 12.8825 & 8.7257 & 4.2245 & 1.6237 & 2.1296 & 1.4764 & 2.0655 \\
\hline
\end{tabular}

gas disk, planets will undergo type I or II migration until they are halted at the inner region of the system which is close to their nominal locations. Finally, their orbits will be circularized owing to the tidal effect raised by the central star.

In this formation scenario, the density profile of gas disk, especially in the hole, is an important factor that affects the final configuration. Usually holes in the gas disk appear at two locations. One is the result of the coupling of the gas with the magnetic field of the star which is related to the star magnetic field $\left(B_{*}\right.$, Koenigl (1991)). Another hole is the boundary between the active zone and the dead zone in the midplane of the gas disk. This boundary is responding to the magnetorotational instability and changes with the star accretion rate $(\dot{M}$, Kretke et al. (2009)). Another important factor that affects the final configuration of the system is the rate of orbital migration particularly the type I migration. Under assumption of linear analysis, type I migration is so fast that does not allow terrestrial planets to form. Several theories have been proposed to reduce the rate of migration or even reverse the migration direction (Kley et al. 2009; Wang \& Zhou 2011).

\section{1. $K O I-152$}

We test the effect of different density profiles and rates of type I migration on the configuration formation of KOI-152 through N-body simulation (Wang et al. (2012)). Figure 1 shows the main results. Panel (a) shows the density profile with different star accretion rates. DM1 and DM2 represent the locations of the inner hole and the boundary between the active zone and dead zone, respectively. The green line and red line express the profile with different star magnetic filed (green: $B_{*}=0.5 \mathrm{KG}$ and red: $B_{*}=2.5 \mathrm{KG}$ ) but same star accretion rate.

For KOI-152, we find that planets are not massive enough to open a gap in the gas disk. Therefore, they all undergo type I migration during the formation process, and their masses are estimated to be in the range of (9-15) $M_{\oplus},(9-19) M_{\oplus}$, and $(20-24)$ $M_{\oplus}$, respectively. Low star accretion rate with $\dot{M} \leqslant 5 \times 10^{-9} M_{\odot} \mathrm{yr}^{-1}$ and low rate of type I migration with one-tenth of the linear analysis results are propitious to form the configuration of KOI-152. Panel (b) and (c) show two cases that can form a configuration 

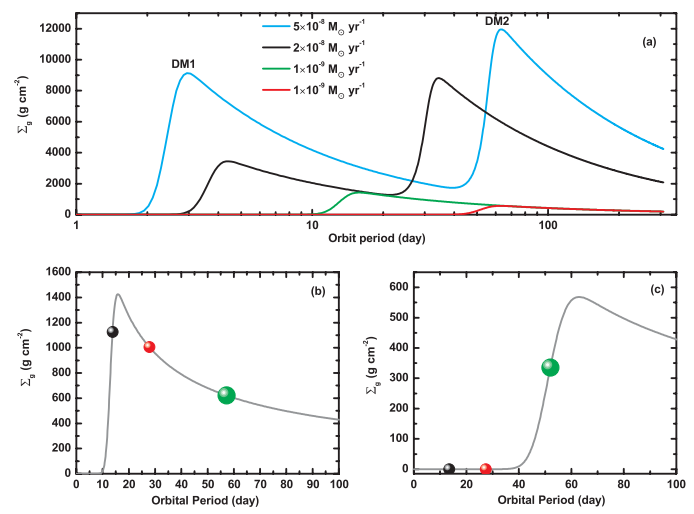

Figure 1. The density profile of gas disk and the final configuration of KOI-152. Panel (a) shows the gas density profile with different star accretion rate. panel (b) and (c) display two cases that can form a configuration similar to KOI-152.

similar to KOI-152. Panel (b) shows the results of the case with $\dot{M}=1 \times 10^{-9} M_{\odot} \mathrm{yr}^{-1}$, $B_{*}=0.5 \mathrm{KG}$ and finally the innermost planet is halted at the location of DM (DM1 and DM2 combine to one) leading other two planets trapped into MMRs with it, while Panel (c) shows the case of $\dot{M}=1 \times 10^{-9} M_{\odot} \mathrm{yr}^{-1}, B_{*}=2.5 \mathrm{KG}$ and finally the outmost planet is stopped at DM resulting the formation of 4:2:1 MMRs configuration. It is worth to mention that the tidal raised by the star will destroy the resonant configuration leading a configuration near 4:2:1 MMRs which is similar to KOI-152.

\subsection{Solar-like system}

In this section in order to get the conditions that lead to the different resonant configurations, such as 3:2 and 2:1 MMRs, we consider a system with a solar-like star and use the same model as in section 2.1. We have obtained results after 20 runs with different $\dot{M}$ (in the range of $\left[1 \times 10^{-9} M_{\odot} \mathrm{yr}^{-1}, 2.5 \times 10^{-8} M_{\odot} \mathrm{yr}^{-1}\right]$ ) and rate of type I migration $([0.01,1]$ times of the linear analysis result). Three out of 40 planet pairs are trapped into 3:2 MMR at the end of the simulations, while 7 out of 40 planet pairs are in 2:1 MMR. From the simulation results we find that two-planet capture in 3:2 MMR i easier when the migration speed is higher than 0.1 times of linear analysis result, or the star accretion rate is not lower than $5 \times 10^{-9} M_{\odot} \mathrm{yr}^{-1}$. The high star accretion rate implies that the planet pair forms at the early stage of the star evolution.

\section{Summary}

Our scenario is successful to explain the configuration formation of KOI-152. Other ten systems shown in Table 1 with similar configuration may bear the same formation scenario. In the early stage of star evolution, 3:2 MMR is more easily occurred, while 2:1 MMR is more likely to happen at the late stages. Since statistically planet pairs near 3:2 MMR are more than 2:1 MMR, one might conclude that the Kepler planet candidates may form at the early stage of star formation, more often.

\section{Acknowledgements}

This work is supported by NSFC (Grants No. 11203087, 11273068, 10925313, 10833001), the Natural Science Foundation of Jiangsu Province (Grant No. BK2009341), the Foundation of Minor Planets of Purple Mountain Observatory, and the innovative and interdisciplinary program by CAS (Grant No. KJZD-EW-Z001). 


\section{References}

Batalha, N. M., Rowe, J. F., Bryson, S. T., et al. 2012, ApJS, 204, article id. 24 Fabrycky, D. C., Lissauer, J. J., Ragozzine, D., et al. 2012, arXiv:1202.6328

Lissauer, J. J., Ragozzine, D., Fabrycky, D. C., et al. 2011, ApJS, 197, 8

Koenigl, A. 1991, ApJL, 370, L39

Kley, W., Bitsch, B., \& Klahr, H. 2009, A\&A, 506, 971

Kretke, K. A., Lin, D. N. C., Garaud, P., \& Turner, N. J. 2009, ApJ, 690, 407

Wang, S. \& Zhou, J. L. 2011, ApJ, 727, 108

Wang, S., Ji, J. H., \& Zhou, J. L. 2012, ApJ, 753, 170 